\title{
Antisparsity as a Prior in Blind Separation of Correlated Sources
}

This paper was downloaded from TechRxiv (https://www.techrxiv.org).

LICENSE

CC BY 4.0

SUBMISSION DATE / POSTED DATE

15-06-2021 / 24-06-2021

CITATION

Brotto, Renan; Nose-Filho, Kenji; Romano, João M. T. (2021): Antisparsity as a Prior in Blind Separation of Correlated Sources. TechRxiv. Preprint. https://doi.org/10.36227/techrxiv.14787684.v1

DOI

10.36227/techrxiv.14787684.v1 


\title{
Antisparsity as a Prior in Blind Separation of Correlated Sources
}

\author{
Renan D. B. Brotto, Kenji Nose-Filho, João M. T. Romano
}

\begin{abstract}
This letter introduces the concept of antisparse Blind Source Separation (BSS), proposing a suitable criterion based on the $\ell_{\infty}$ norm to explore the antisparsity feature. The effectiveness of the criterion is theoretically demonstrated and it is also evaluated by computational simulations, which consider up to ten distinct sources with different correlation levels. Moreover, we simulated a scenario in wireless communication with binary sources, comparing our approach to the Constant Modulus algorithm. Both the theoretical and the simulation results highlight the potentiality of using antisparsity as a prior in BSS.
\end{abstract}

Index Terms-Antisparsity, Blind Source Separation, Correlated Sources, Unsupervised Signal Processing.

\section{INTRODUCTION}

The problem of Blind Source Separation (BSS) [1], [2] consists in recovering a set of signals, called sources, which have been combined by a mixing system. The separation process is usually based on recovering some prior information about the signals, i.e., a feature that precisely describes the sources. Two important priors that have been extensively studied in the literature are the statistical independence between the sources, which is related to the classical Independent Component Analysis (ICA) [3], [4], and the sources sparsity, which inaugurated the Sparse Component Analysis (SCA) [5], [6], [7].

This letter presents and analyzes an alternative prior information that, to the best of our knowledge, has not been explored in BSS: the antisparsity. In a dual way to the case of sparse sources, the notion of antisparsity is associated with the democratic or equitable distribution. So, an antisparse signal tends to spread its energy uniformly among its samples and such property can be evaluated by means of the signal $\ell_{\infty}$ norm [8].

The next section presents the main contribution of this work: the proposition of an antisparsity-based criterion for BSS and the demonstration of its effectiveness. It is worth pointing out that it does not depend on the sources correlation level. Section III provides several simulation results, varying the number of sources and the correlation level, in order to assess the performance of the proposed approach. We also

Letter submitted at June, $15^{\text {th }}$ 2021. The authors would like to thank FAPESP (processes number 2017/13025-2 and 2019/20899-4) and CAPES, Code 001.

Renan D. B. Brotto and João M. T. Romano are with the School of Electrical and Computer Engineering, University of Campinas, Campinas, São Paulo, Brazil (e-mail: rbrotto@decom.fee.unicamp.br, romano@decom.fee.unicamp.br).

Kenji Nose-Filho is with Center of Engineering, Modeling and Applied Social Sciences, Federal University of ABC (UFABC), Santo André, São Paulo, Brazil (e-mail: kenji.nose@ufabc.edu.br). consider in our simulations the recovery of binary sources in a wireless communication system, highlighting the potentiality of the antispasity application in real scenarios. Finally, Section IV briefly sets out our conclusions and perspectives of future works.

\section{THE PROPOSED ANTISPARSITY-BASED CRITERION FOR BSS}

The process of linear and instantaneous mixing, considering the case of determined mixtures (number of mixtures equal to the number of sources), is given by:

$$
\mathbf{X}=\mathbf{H S},
$$

where

$$
\mathbf{S}=\left[\begin{array}{cccc}
s_{1}(0) & s_{1}(1) & \cdots & s_{1}(T-1) \\
s_{2}(0) & s_{2}(1) & \cdots & s_{2}(T-1) \\
\vdots & \vdots & \ddots & \vdots \\
s_{N}(0) & s_{N}(1) & \cdots & s_{N}(T-1)
\end{array}\right]=\left[\begin{array}{c}
\mathbf{s}_{1} \\
\mathbf{s}_{2} \\
\vdots \\
\mathbf{s}_{N}
\end{array}\right]
$$

and

$$
\mathbf{X}=\left[\begin{array}{cccc}
x_{1}(0) & x_{1}(1) & \cdots & x_{1}(T-1) \\
x_{2}(0) & x_{2}(1) & \cdots & x_{2}(T-1) \\
\vdots & \vdots & \ddots & \vdots \\
x_{N}(0) & x_{N}(1) & \cdots & x_{N}(T-1)
\end{array}\right]=\left[\begin{array}{c}
\mathbf{x}_{1} \\
\mathbf{x}_{2} \\
\vdots \\
\mathbf{x}_{N}
\end{array}\right]
$$

denote, respectively, the matrices containing all of the sources and mixtures data, considering $T$ available samples. The matrix $\mathbf{H} \in \mathbb{R}^{\mathrm{N} \times \mathrm{N}}$ performs the mixing process.

In order to appropriately estimate the sources, we look for a separating system $\mathbf{W} \in \mathbb{R}^{\mathrm{N} \times \mathrm{N}}$, leading to:

$$
\hat{\mathbf{S}}=\mathbf{W X}
$$

where the estimates data matrix $\hat{\mathbf{S}}$ is defined in a similar way as expressed in equations (2) and (3) for matrices $\mathbf{S}$ and $\mathbf{X}$.

For statistical independent sources the process of source separation is usually done in two steps. In the first one, we perform a whitening pre-processing, by multiplying the mixtures $\mathbf{X}$ with a whitening matrix $\mathbf{P}$ typically obtained by a PCA related technique [1] [3]. With the decorrelated sources $\mathbf{X}^{\prime}=\mathbf{P X}$, the second step consists in optimizing a rotation matrix $\mathbf{U}$ with a suitable criterion, intrinsically related to the prior information. So that we can write the estimated sources as $\hat{\mathbf{S}}=\mathbf{U X}^{\prime}$. In this work, we explore the antisparsity and evaluate it with the $\ell_{\infty}$ norm, leading to the following optimization problem: 


$$
\max _{\mathbf{U}} \frac{1}{\left\|\mathbf{U X}^{\prime}\right\|_{\infty}^{2}}
$$

where the associated matrix norm is defined by

$\left\|\mathbf{U X}^{\prime}\right\|_{\infty}=\sum_{i=1}^{N}\left\|\mathbf{u}_{i} \mathbf{X}^{\prime}\right\|_{\infty}=\sum_{i=1}^{N}\left\|\hat{\mathbf{s}}_{i}\right\|_{\infty}=\sum_{i=1}^{N} \max _{n}\left(\left|\hat{s}_{i}(n)\right|\right)$.

In (6), $\mathbf{u}_{i}$ denotes the $i$-th row of the rotation matrix, and $\|\cdot\|_{\infty}$ is the $\ell_{\infty}$ norm. Hence, for our purposes here, the norm taken over the estimates matrix $\hat{\mathbf{S}}=\mathbf{U X}^{\prime}$ corresponds to the sum of the $\ell_{\infty}$ norms over each source estimate $\hat{\mathbf{s}}_{\mathbf{i}}=$ $\mathbf{u}_{i} \mathbf{X}^{\prime}, i=1,2, \cdots, N$.

Since $\mathbf{P}$ is the PCA matrix, we can write the result of the whitening step as

$$
\mathbf{P H}=\mathbf{V} \boldsymbol{\Lambda},
$$

where $\boldsymbol{\Lambda} \in \mathbb{R}^{\mathrm{N} \times \mathrm{N}}$ is a diagonal matrix and $\mathbf{V} \in \mathbb{R}^{\mathrm{Nx} N}$ is another rotation matrix.

So, from equations (1) and (7), we can rewrite the estimates as follows:

$$
\hat{\mathbf{S}}=\mathbf{U V} \boldsymbol{\Lambda} \mathbf{S}
$$

which corresponds to the global process of mixing and separation.

Since the BSS problem admits a scale ambiguity, we can neglect the effect of $\boldsymbol{\Lambda}$. Then, because the product of rotation matrices is another rotation matrix, we can combine $\mathbf{U}$ and $\mathbf{V}$ into a equivalent rotation matrix $\mathbf{U}_{G}$ :

$$
\hat{\mathbf{S}}=\mathbf{U}_{G} \mathbf{S}
$$

For the sake of simplicity, let us first consider the case of two sources, $s_{1}(n)$ and $s_{2}(n)$, with the associated estimates $\hat{s}_{1}(n), \hat{s}_{2}(n)$. Let us also consider antisparse sources, with uniform distribution, zero mean and supports $\left[-A_{1}, A_{1}\right]$ and $\left[-A_{2}, A_{2}\right]$, respectively. Therefore, we have:

$$
\max _{n}\left(\left|s_{1}(n)\right|\right)=A_{1}, \max _{n}\left(\left|s_{2}(n)\right|\right)=A_{2} .
$$

Particularly for the case of two sources, we can write $\mathbf{U}_{G}$ as:

$$
\mathbf{U}_{G}=\left[\begin{array}{cc}
\cos \theta_{G} & \sin \theta_{G} \\
-\sin \theta_{G} & \cos \theta_{G}
\end{array}\right] .
$$

Then, from (9) and (11) we can explicitly relate the estimates and the sources:

$$
\begin{aligned}
& \hat{s}_{1}(n)=\cos \theta_{G} s_{1}(n)+\sin \theta_{G} s_{2}(n), \\
& \hat{s}_{2}(n)=-\sin \theta_{G} s_{1}(n)+\cos \theta_{G} s_{2}(n) .
\end{aligned}
$$

To optimize the separation matrix, we employ the proposed criterion in (5), leading to

$$
J\left(\theta_{G}\right)=\frac{1}{\left\|\hat{\mathbf{s}}_{1}\right\|_{\infty}^{2}+\left\|\hat{\mathbf{s}}_{2}\right\|_{\infty}^{2}},
$$

where

$$
\begin{aligned}
\left\|\hat{\mathbf{s}}_{1}\right\|_{\infty}^{2} & =\left(\max \left(\left|\cos \theta_{G} s_{1}(n)+\sin \theta_{G} s_{2}(n)\right|\right)\right)^{2} \\
& =A_{1}^{2} \cos ^{2} \theta_{G}+2 A_{1} A_{2}\left|\cos \theta_{G} \sin \theta_{G}\right|+A_{2}^{2} \sin ^{2} \theta_{G}
\end{aligned}
$$

and

$$
\left\|\hat{\mathbf{s}}_{2}\right\|_{\infty}^{2}=A_{1}^{2} \sin ^{2} \theta_{G}+2 A_{1} A_{2}\left|\cos \theta_{G} \sin \theta_{G}\right|+A_{2}^{2} \cos ^{2} \theta_{G} .
$$

Combining (14) and (15) we have:

$$
\left\|\hat{\mathbf{s}}_{1}\right\|_{\infty}^{2}+\left\|\hat{\mathbf{s}}_{2}\right\|_{\infty}^{2}=A_{1}^{2}+4 A_{1} A_{2}\left|\sin \theta_{G} \cos \theta_{G}\right|+A_{2}^{2},
$$

which can be rewritten as

$$
\left\|\hat{\mathbf{s}}_{1}\right\|_{\infty}^{2}+\left\|\hat{\mathbf{s}}_{2}\right\|_{\infty}^{2}=A_{1}^{2}+2 A_{1} A_{2}\left|\sin 2 \theta_{G}\right|+A_{2}^{2} .
$$

Therefore, $J\left(\theta_{G}\right)$ becomes:

$$
J\left(\theta_{G}\right)=\frac{1}{A_{1}^{2}+A_{2}^{2}+2 A_{1} A_{2}\left|\sin 2 \theta_{G}\right|} .
$$

Since $\left|\sin 2 \theta_{G}\right| \in[0,1]$, the maximum of $J\left(\theta_{G}\right)$ occurs for $\sin 2 \theta_{G}=0$. So, we have two possible solutions for $\theta \in[0, \pi[$ :

1) $\theta_{G}=0$, which leads to $\hat{s}_{1}(n)=s_{1}(n)$ and $\hat{s}_{2}(n)=$ $s_{2}(n)$,

2) $\theta_{G}=\frac{\pi}{2}$, where we have $\hat{s}_{1}(n)=s_{2}(n)$ and $\hat{s}_{2}(n)=$ $-s_{1}(n)$.

In 1) and 2), we can verify that the maximum points of $J\left(\theta_{G}\right)$ correspond to the sources separation, possibly with a permutation and a sign inversion. Thus, the antisparsity, measured here by the $\ell_{\infty}$ norm, can be used as a prior in the BSS problem.

It is interesting to note that if $\mathbf{H}$ is just a rotation matrix, then the algebraic development made from (9) still holds not only for statistical independent sources but also for correlated ones, indicating the potential use of the antisparsity in this scenario.

\section{Generalizing the Proposed Criterion to $N$ Sources}

So far, we have demonstrated that our criterion is sufficient for two sources and now we are going to extend it to the case of $N$ signals, by decomposing a multidimensional rotation as the combination of two-dimensional ones. To better clarify this point, let us consider the case of three sources, just for visual purposes. Once the mixtures have been whitened, we must align the estimates directions with those of the sources. This is carried out by a rotation process, as previously discussed and illustrated in Fig. 1a. 


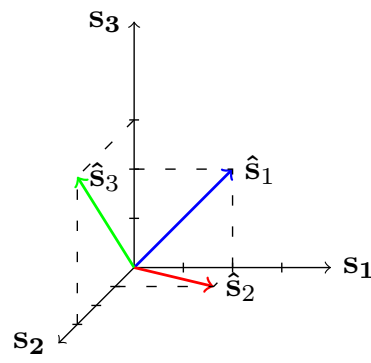

(a) Before rotation.

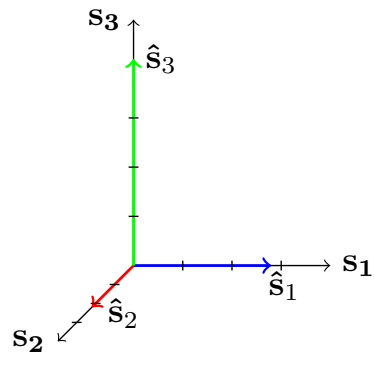

(b) After rotation.
Fig. 1: Visual representation of the separation process, with sources already decorrelated.

Let us consider the estimate $\hat{\mathbf{s}}_{1}$. To align it with the source $\mathbf{s}_{1}$ (and here we are assuming that there is no permutation) we must perform a rotation in the 3D space. This rotation can be decomposed in three successive two-dimensional ones: the first one performed in the $\mathbf{s}_{1}-\mathbf{s}_{2}$ plane; the second one in the $\mathbf{s}_{1}-\mathbf{s}_{3}$ plane; and the last one in the $\mathbf{s}_{2}-\mathbf{s}_{3}$ plane. Therefore, for the case of three sources, we can proceed with the same analysis as previously done, but repeated three times, one for each plane. Once all the rotations were performed, the sources will be recovered, as shown in Fig. $1 \mathrm{~b}$.

The generalization to the case of $N$ sources is straightforward: we carry out $N(N-1) / 2$ two-dimensional rotations, one for each $\mathbf{s}_{i}-\mathbf{s}_{j}$ plane, $i=1,2, \cdots N, j=i+1, \cdots N$. Such procedure can be achieved by means of the Givens rotations method [9], [10].

With the generalization of our previous analysis from 2 to $N$ sources made, in the next section we present the simulation results about the use of the antisparsity as a prior in BSS.

\section{Simulation Results}

In this section we present the simulation results with antisparse sources, including the case of correlated signals. For that we consider a random mixing system $\mathbf{H}$ that only rotates the sources. For statistical independent sources it is equivalent to assume that the whitening step has been previously done. We adjust $\mathbf{W}$ with the Givens rotations method, so we can decompose a rotation effect in a multidimensional space into the combination of rotations in two-dimensional spaces.

For $N$ sources, we obtain the optimal rotation angle $\beta_{i}$, $i=1,2, . ., N(N-1) / 2$ for each considered plane, using the golden section method [11] in the interval $[0, \pi / 2]$, to maximize (5).

To evaluate the proposed method performance, we use the signal-to-interference ratio (SIR) between the estimates $\hat{s}_{i}(n)$ and sources $s_{i}(n)$ :

$$
\operatorname{SIR}_{\mathrm{dB}}\left(\hat{\mathbf{s}}_{j}\right)=\max _{i}\left(10 \log \left(\frac{\left\|\mathbf{s}_{i}\right\|^{2}}{\left\|\mathbf{s}_{i}-\hat{\mathbf{s}}_{j}\right\|^{2}}\right)\right) .
$$

Since in the Blind Source Separation problem we may have a sign ambiguity in the estimates, we must evaluate $\operatorname{SIR}_{d B}\left(\hat{\mathbf{s}}_{j}\right)$ and $\operatorname{SIR}_{d B}\left(-\hat{\mathbf{s}}_{j}\right)$, and take the maximum value between them.

In all the simulations, we consider zero mean sources, with unitary variance and uniform distribution. To introduce dependence among the sources, we apply a correlation level $\alpha$ to any pair of signals, such that:

$$
\mathbb{E}\left[s_{i}(n) s_{j}(n)\right]= \begin{cases}1 & \text { if } i=j, \\ \alpha & \text { if } i \neq j .\end{cases}
$$

In the first simulation, we considered independent sources and compared the proposed method with the Fast ICA [12] algorithm, without a previous whitening pre-processing. We show the results in Figure 2.

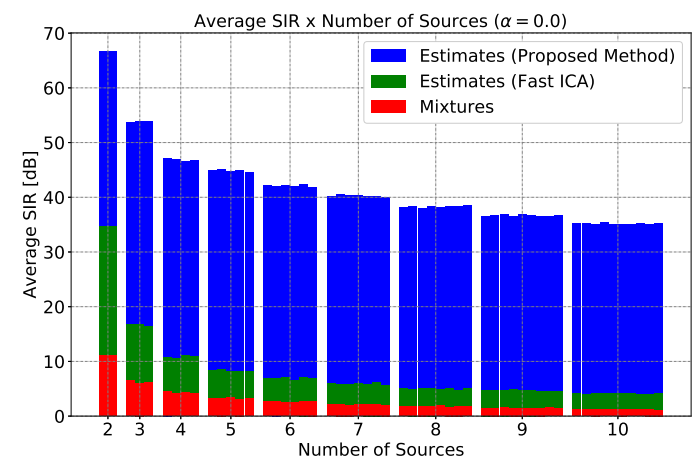

Fig. 2: Comparative of average SIR for decorrelated sources.

In Fig. 2 we can observe the average SIR level, taken from 500 Monte Carlo simulations, as a function of $N$. The blue bars represent the SIR level of the estimates obtained by the proposed method, while the green ones depict the SIR level using Fast ICA algorithm. For comparative purposes, we have also presented the SIR level of the mixtures, in red.

We observe that the proposed method outperforms the Fast ICA algorithm for $N=2,3, \cdots, 10$ sources, reaching a SIR level above $35 \mathrm{~dB}$ level in all cases. It is worth pointing out that when we assume both independence and antisparsity priors, the proposed method provides better results than an ICA-based one.

In our second simulation, we considered sources with correlation level $\alpha=0.5^{1}$, with the results shown in Fig. 3. From these results, we can observe the robustness of the proposed method, since it is able to recover up to 10 correlated sources, outperforming, again, the Fast ICA method for all $N$ evaluated. Also, it is important to note that the Fast ICA algorithm had an inferior performance (in terms of SIR) than that obtained by the mixtures, indicating, as expected, that this method is not suitable for correlated signals.

Finally, we evaluated the antisparsity in the recovery of binary sources ${ }^{2}$, which are very common in the context of wireless communications [13]. We compared the proposed method with the Constant Modulus Algorithm, precisely the Cross-Correlation Multi-Modulus Algorithm (CC- $\beta$ MMA), with $\beta=1$, presented in [14], a very suitable one for

\footnotetext{
${ }^{1}$ To generate correlated antisparse sources we first generated correlated Gaussian ones. Then we applied the Cumulative Distribution Function (CDF) to obtain uniform sources with the desired correlation level. Finally we extracted the mean and appropriately scaled the sources.

${ }^{2}$ In this case, we repeated the same process used to generate uniform sources and then we applied a decision rule in order to obtain symbols \pm 1 .
} 


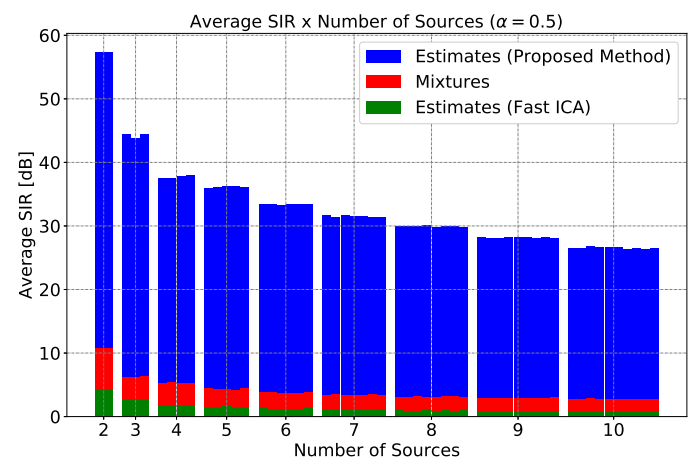

Fig. 3: Comparative of average SIR for correlated sources $(\alpha=0.5)$.

this kind of sources. Again, we generated random rotation mixing systems and used the Symbol Error Rate (SER) as a performance measure. In the first case, depicted in Fig. 4, we have considered independent signals, hence $\alpha=0$, varied the number of sources $(N=2$ in blue lines, $N=5$ in orange lines and $N=7$ in red lines) and the Signal-to-noise ratio (SNR) in the range $[30 \mathrm{~dB}, 20 \mathrm{~dB}, 10 \mathrm{~dB}]$, with Gaussian noise. The performance of our approach, i.e., the mean SER taken from 500 Monte Carlo simulations, is represented by filled squares and that of CC- $\beta$ MMA algorithm is represented by filled circles.

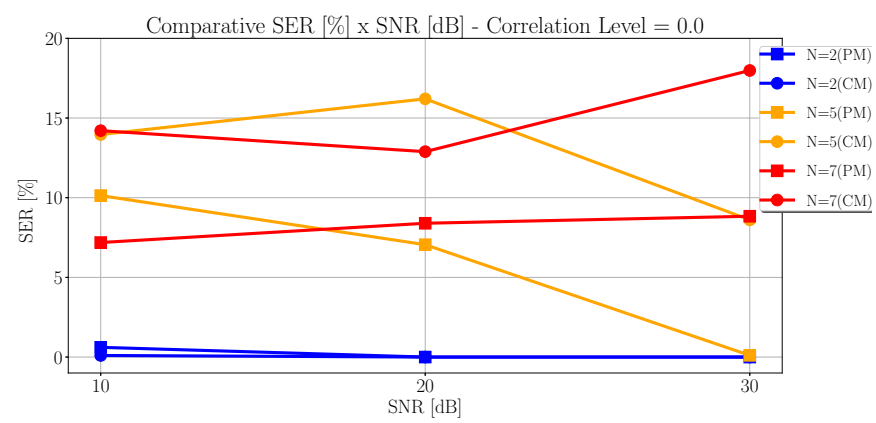

Fig. 4: SER obtained with the proposed method (PM) and Constant Modulus (CM) algorithm for independent sources, considering different noise levels.

From Fig. 4, we can observe that for two sources our approach presented a performance very close to that of the $\mathrm{CM}$, with a little higher SER when SNR $=10 \mathrm{~dB}$. For $N=5,7$, our method has led to a better performance, and the proposed method has obtained a SER of at most $10 \%$ for all SNRs considered. Besides, our method presented a performance very similar for 5 and 7 sources (especially in low SNR scenarios), which suggests a robustness of our approach with respect to the number of signals to be recovered. We also note some abnormal behaviors, since we can observe little improvements in the SER level even when we reduced the SNR ratio; this is due to the statistical variability, caused by the limited number of simulations, and does not compromise the previous analysis.
We repeated the experiment for correlated binary sources $(\alpha=0.5)$, as shown in Fig. 5 .

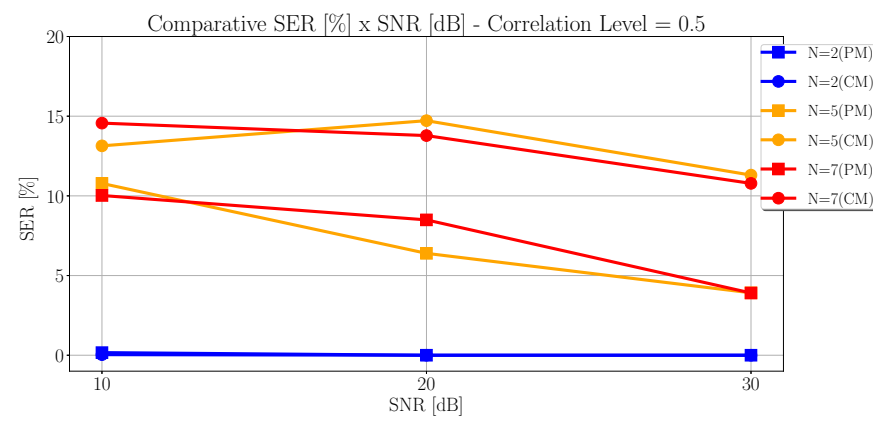

Fig. 5: SER obtained with the proposed method (PM) and Constant Modulus (CM) algorithm for correlated sources $(\alpha=$ $0.5)$, considering different noise levels.

As occurred in the case of independent sources, our approach had a close performance to the CM method for $N=2$. For the other cases, we can observe the robustness of our approach with respect to the number of sources: for $N=5$ and $N=7$ the antisparsity outperformed the CM method and our approach led to a very similar SER level for these number of sources, both limited by a $10 \%$ rate. As we stated in the previous simulation, the statistical variability observed does not compromise the analysis made.

\section{CONCLUSION}

In this work we have introduced the antisparsity as a prior information to Blind Source Separaion. We have developed a criterion, based on the $\ell_{\infty}$ norm, and provided a theoretical demonstration of its effectiveness. The proposed method is capable of recovering up to 10 sources, even when they are correlated.

We evaluated our approach in a wireless communication scenario, recovering binary sources with low SER level for different SNRs. As we can observe, our approach outperformed the Constant Modulus algorithm in most of the scenarios highlighting the potential use of the antisparsity in telecommunication problems. Since CM algorithm is related to the techniques of Bounded Component Analysis (BCA) [15], a relatively recent and very interesting topic in the Blind Source Separation area, the results presented in this letter open the possibility of exploring, in future works, the relationship between the antisparsity and the BCA techniques, both theoretically and in real world applications.

We are also interested in investigating the development of a more general framework, able to handle correlated sources for general mixing systems.

\section{REFERENCES}

[1] P. Comon and C. Jutten, eds., Handbook of blind source seapartion. Academic Press, 2010.

[2] J. M. T. Romano, R. Attux, C. C. Cavalcante, and R. Suyama, Unsupervised signal processing: channel equalization and source separation. CRC Press, 2011. 
[3] A. Hyvarinen, J. Karhunen, and E. Oja, Independent component analysis. John Wiley \& Sons, 2001.

[4] P. Comon, "Independent component analysis, a new concept?," Signal Processing, vol. 36, no. 3, pp. 287-314, 1994.

[5] R. Gribonval and S. Lesage, "A survey of sparse component analysis for blind source separation: principles, perspectives, and new challenges," in Proceedings of the 2006 ESANN, (Bruges, Belgium), pp. 323-330, 2006.

[6] K. Nose-Filho and J. M. T. Romano, "Low-rank decomposition based on disjoint component analysis with applications in seismic imaging," IEEE Transactions on Computational Imaging, vol. 3, pp. 275-281, 2017.

[7] C. Chang, P. Fung, and Y.S.Hung, "On a sparse component analysis approach to blind source separation," Rosca J., Erdogmus D., Príncipe J.C., Haykin S. (eds) Independent Component Analysis and Blind Signal Separation, vol. 3889, pp. 765-772, 2006.

[8] C. Elvira, P. Chainais, and N. Dobigeon, "Bayesian antisparse coding," IEEE Transaction on Signal Processing, vol. 65, pp. 1660-1672, 2017. DOI:https://doi.org/https://doi.org/10.1109/TSP.2016.2645543.

[9] G. H. Golub and C. F. Van Loan, Matrix Computations. John Hopkins, 3rd ed., 1996.

[10] S. A. W. Shah, K. Abed-Meraim, and T. Y. Al-Naffouri, "Blind source separation algorithms using hyperbolic and givens rotations for highorder qam constellations," IEEE Transactions on Signal Processing, vol. 66, no. 7, pp. 1802-1816, 2018.

[11] W. H. Press, S. A. Teukolsky, W. T. Vetterling, and B. P. Flannery, Numerical recipes: the art of scientific computing. Cambridge, 2007.

[12] A. Hyvarinen, "Fast and robust fixed-point algorithms for independent component analysis," IEEE Transactions on Neural Networks, vol. 10, pp. 626-634, 1999.

[13] Z. Luo, C. Li, and L. Zhu, "A comprehensive survey on blind source separation for wireless adaptive processing: Principles, perspectives, challenges and new research directions," IEEE Access, vol. 6, pp. 6668566708, 2018.

[14] W. Cong, L. Yang, C. Sun, R. Yang, and S. Zhu, "Blind source separation and equalization for high order qam signals in mimo system," in 2018 10th International Conference on Intelligent Human-Machine Systems and Cybernetics (IHMSC), vol. 02, pp. 52-55, 2018.

[15] S. Cruces, "Bounded component analysis of linear mixtures: A criterion of minimum convex perimeter," IEEE Transactions on Signal Processing, vol. 58, no. 4, pp. 2141-2154, 2010. 